Preprint de "Weak stability and a bargaining set for the marriage model," de Flip Klijn i Jordi Massó. Games and Economic Behavior 42, 91-100 (2003). Lliurat a Elsevier el gener de 2002. 


\title{
Weak Stability and a Bargaining Set for the Marriage Model ${ }^{1}$
}

\author{
Flip Klijn² and Jordi Massó 3 \\ Departament d'Economia i d'Història Econòmica and CODE, \\ Universitat Autònoma de Barcelona, 08193 Bellaterra (Barcelona), Spain. \\ E-mails: fklijn@pareto.uab.es and jordi.masso@uab.es
}

January 2002

Running title: Matching: Weak Stability and a Bargaining Set

\footnotetext{
${ }^{1}$ We thank Herbert Hamers, an anonymous referee, and an Associate Editor for their useful comments.

${ }^{2}$ To whom correspondence should be addressed. Part of this research was carried out while this author was $\mathrm{Ph} . \mathrm{D}$. student at the Department of Econometrics and CentER, Tilburg University.

${ }^{3}$ This author's work is partially supported by Research Grants DGES PB98-0870 from the Dirección General de Investigación Científica y Técnica (Spanish Ministry of Education and Culture) and 2000SGR-00054 from the Departament d'Universitats, Recerca i Societat de la Informació (Generalitat de Catalunya).
} 


\begin{abstract}
In this note we introduce weak stability, a relaxation of the concept of stability for the marriage model by assuming that the agents are no longer myopic in choosing a blocking pair. The new concept is based on threats within blocking pairs: an individually rational matching is weakly stable if for every blocking pair one of the members can find a more attractive partner with whom he forms another blocking pair for the original matching. Our main result is that under the assumption of strict preferences, the set of weakly stable and weakly efficient matchings coincides with the bargaining set of Zhou (1994) for this context.
\end{abstract}

Keywords: matching, (weak) stability, bargaining set

Journal of Economic Literature Classification Number: C78 


\section{Introduction}

In matching models, stability is one of the subjects that have been studied most. A steady basis was laid by Gale and Shapley (1962), who formalized the notion of stability and who in addition provided an algorithmic way to calculate stable matchings in their marriage model. A matching is stable if all agents have acceptable spouses and there is no unmarried couple whose members both like each other better than their current spouses. The paper of Gale and Shapley (1962) has led to a vast body of literature in which the properties of the set of stable matchings were studied, not only for the marriage model, but for more general models as well. We refer to Roth and Sotomayor (1990) for a comprehensive account on the study of the set of stable matchings.

Rather than studying the set of stable matchings in yet another model, we have opted for weakening the concept of stability in the marriage model. We were led to this by the observation that a blocking pair, which undermines the stability of a matching, is not always credible in the sense that one of the partners may find a more attractive partner with whom he forms another blocking pair for the original matching. We enlarge the set of stable matchings by allowing for this kind of blocking pairs. In other words, we define an individually rational matching to be weakly stable if every blocking pair is - in the sense above - not credible. Clearly, weak stability is a relaxation of stability. In this view, the paper of Gärdenfors (1975) should be mentioned here. From the perspective of voting theory, he defined a matching to be a majority assignment if there is no other matching that is preferred by a majority (of men and women) to the original matching. Gärdenfors observed that, when preferences are strict, the set of majority assignments comprises the set of stable matchings, thus showing that the notion of majority assignment is a relaxation of stability. To make comparisons between different matchings, Gärdenfors assumed the two sides of the marriage market to have the same cardinality. We do not need to make this assumption, because our concept is solely related to the credibility of blocking pairs. A more serious drawback of the concept of majority assignment is that in certain situations it fails to rule out matchings with a unique blocking pair. Our relaxation, however, excludes this kind of matchings, and we think that it is a minimal condition that a 'good' relaxation should satisfy.

Not surprisingly, the nice lattice structure of the set of stable matchings does not carry over to the set of weakly stable matchings. In fact, we will see that the decomposition lemma of Knuth (1976) does not longer hold for the set of weakly stable matchings. The decomposition lemma was a key result to show, among others, the lattice structure of the set of stable matchings.

Nevertheless, we are able to present a result similar to the equality of the core and the set of stable matchings in a marriage market. We translate the bargaining set of Zhou (1994) to the marriage model and prove that it coincides with the set of weakly stable matchings that are weakly efficient. A matching is weakly efficient if there is no other matching in which all agents are better off. We need the condition of weak efficiency, since, following Zhou (1994), we do not allow for counterobjections against objections of the set of all agents. So, all matchings in the bargaining set are weakly efficient, whereas weakly stable matchings may not be weakly efficient.

The work is organized as follows. In Section 2 we recall the marriage model of Gale and Shapley (1962), define the concept of weak stability, and give the definition of the bargaining set of Zhou (1994) for the marriage model. In Section 3 we study the structure of the set of 
weakly stable matchings. Then, in Section 4, we link the concept of weak stability with Zhou's (1994) bargaining set. Some concluding remarks follow in Section 5.

\section{Notation and definitions}

In this section we recall the marriage model of Gale and Shapley (1962). Most of the notation is due to Roth and Sotomayor (1990). After that, we define the concept of weak stability. Finally, we give the definition of the bargaining set of Zhou (1994) for the marriage model.

There are two finite and disjoint sets of agents: a set $M=\left\{m_{1}, \ldots, m_{r}\right\}$ of 'men' and a set $W=\left\{w_{1}, \ldots, w_{s}\right\}$ of 'women', where possibly $r \neq s$. Let $N=M \cup W$. We sometimes denote a generic agent by $i$ and a generic man and a generic woman by $m$ and $w$, respectively. Each agent has a complete and transitive preference ordering over the agents on the other side of the market and the prospect of being alone. We assume that all preferences are strict. Hence, the preference of a man $m$ can be represented by a preference list $P(m)$ on $W \cup\{m\}$, for instance:

$$
P(m)=w_{3}, w_{2}, m, w_{1}, w_{4}, \ldots, w_{s}
$$

which indicates that $m$ prefers $w_{3}$ to $w_{2}$ and he prefers remaining single to marrying anyone else. Similarly, the preference of a woman $w$ can be represented by a preference list $P(w)$ on $M \cup\{w\}$. Let $P$ be the set of preference lists:

$$
P=\left\{P\left(m_{1}\right), \ldots, P\left(m_{r}\right), P\left(w_{1}\right), \ldots, P\left(w_{s}\right)\right\} .
$$

A marriage market is a triple $(M, W, P)$. We write $w \succ_{m} w^{\prime}$ if $m$ prefers $w$ to $w^{\prime}$, and $w \succeq_{m} w^{\prime}$ if $m$ likes $w$ at least as well $w^{\prime}$ (we need the notation $\succeq_{m}$ because we will be considering situations where $w$ and $w^{\prime}$ may be the same woman). Similarly we write $m \succ_{w} m^{\prime}$ and $m \succeq_{w} m^{\prime}$. A woman $w$ is acceptable to a man $m$ if $w \succ_{m} m$. Analogously, $m$ is acceptable to $w$ if $m \succ_{w} w$.

An outcome for a marriage market $(M, W, P)$ is a matching, a one-to-one function $\mu$ from $N$ to itself, such that for each $m \in M$ and for each $w \in W$ we have $\mu(m)=w$ if and only if $\mu(w)=m$, and if $\mu(m)$ is not contained in $W$ then $\mu(m)=m$, and similarly $\mu(w)=w$ if $\mu(w)$ is not contained in $M$. If $\mu(m)=w$, then man $m$ and woman $w$ are said to be matched to one another. If $\mu(i)=i$, then agent $i$ is said to be single or unmatched. A matching $\mu$ is individually rational if each agent is acceptable to his or her mate, i.e., $\mu(i) \succeq_{i} i$ for all $i \in N$. For a given matching $\mu$, a pair $(m, w)$ is said to form a blocking pair if they are not matched to one another but prefer one another to their mates at $\mu$, i.e., $w \succ_{m} \mu(m)$ and $m \succ_{w} \mu(w)$. A matching is said to be stable if it is individually rational and if there are no blocking pairs. Gale and Shapley (1962) proved that the set of stable matchings is non-empty.

Next, we introduce our concept of weak stability. We say that a blocking pair $(m, w)$ for a matching $\mu$ is weak if for the man $m$ or the woman $w$ there exists another blocking pair for $\mu$ in which he or she is better off than with $w$ and $m$, respectively. Formally,

Definition 2.1 A blocking pair $(m, w)$ for $\mu$ is called weak if there is a woman $w^{\prime} \in W$ such that $w^{\prime} \succ_{m} w$ and $\left(m, w^{\prime}\right)$ is a blocking pair for $\mu$, or a man $m^{\prime} \in M$ such that $m^{\prime} \succ_{w} m$ and $\left(m^{\prime}, w\right)$ is a blocking pair for $\mu$. 
Definition 2.2 A matching $\mu$ is weakly stable if it is individually rational and if all blocking pairs are weak.

Note that a matching with a single blocking pair cannot be weakly stable. The concept of weak stability reflects the idea that agents are not myopic. Thus, weak blocking pairs are ruled out, since they are not credible in the sense that one of the partners may decide to form another blocking pair, leaving the former blocking pair partner behind with a painful illusion. Let $(m, w)$ be a weak blocking pair. We write $(m, w) \rightarrow\left(m, w^{\prime}\right)$ if $w^{\prime} \in W, w^{\prime} \succ_{m} w$, and $\left(m, w^{\prime}\right)$ is a blocking pair for $\mu$. Similarly, we write $(m, w) \rightarrow\left(m^{\prime}, w\right)$ if $m^{\prime} \in M, m^{\prime} \succ_{w} m$, and $\left(m^{\prime}, w\right)$ is a blocking pair for $\mu$. In the first case we say that blocking pair $\left(m, w^{\prime}\right)$ dominates blocking pair $(m, w)$. In the second case blocking pair $\left(m^{\prime}, w\right)$ dominates blocking pair $(m, w)$. Note that both cases may hold at the same time.

Now, we will define a bargaining set for the marriage model. But first we need some more definitions for expositional convenience. A coalition $S$ is a subset of the set of agents $N$. Given a matching $\mu$, a coalition $S$ is said to be able to enforce a matching $\mu^{\prime}$ over $\mu$, if the following condition holds:

(E) for all $i \in S$, if $\mu^{\prime}(i) \neq \mu(i)$, then $\mu^{\prime}(i) \in S$.

Notice that the concept of enforcement is independent of the preferences. An illustration of the concept of enforcement is given by Example 2.3.

Example 2.3 Let $M=\left\{m_{1}, \ldots, m_{7}\right\}$ and $W=\left\{w_{1}, \ldots, w_{7}\right\}$ be the two sets of agents. Let $\mu$ be the matching defined by $\mu\left(m_{i}\right)=w_{i}$ for all $m_{i} \in M \backslash\left\{m_{7}\right\}, \mu\left(m_{7}\right)=m_{7}$, and $\mu\left(w_{7}\right)=w_{7}$. One readily verifies condition (E) to see that $S=\left\{m_{1}, \ldots, m_{4}, w_{1}, \ldots, w_{6}\right\}$ can enforce the matching $\mu^{\prime}$ defined by $\mu^{\prime}(i)=i$ for $i \in\left\{m_{1}, m_{5}, w_{1}, w_{3}\right\}, \mu^{\prime}\left(m_{2}\right)=w_{2}, \mu^{\prime}\left(m_{3}\right)=w_{4}$, $\mu^{\prime}\left(m_{4}\right)=w_{5}, \mu^{\prime}\left(m_{6}\right)=w_{6}$, and $\mu^{\prime}\left(m_{7}\right)=w_{7}$. See Figure $1 . \diamond$

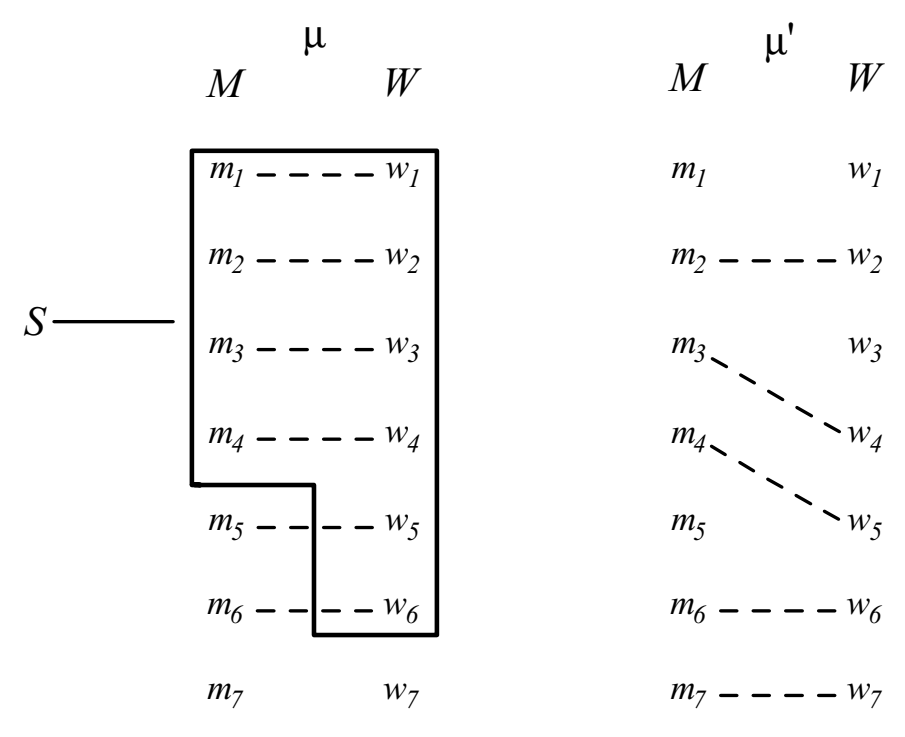

Figure 1: Coalition $S$ enforces the matching $\mu^{\prime}$ over $\mu$ 
Following Zhou (1994) we now define the concepts of objection, counterobjection, and bargaining set for the marriage model.

Definition 2.4 An objection against a matching $\mu$ is a pair $\left(S, \mu^{\prime}\right)$ where $S$ is a coalition and $\mu^{\prime}$ a matching that can be enforced over $\mu$ by $S$ and in which all agents in $S$ are better off than in $\mu$, i.e., it holds that $\mu^{\prime}(i) \succ_{i} \mu(i)$ for all $i \in S$.

Definition 2.5 A counterobjection against an objection $\left(S, \mu^{\prime}\right)$ is a pair $\left(T, \mu^{\prime \prime}\right)$ where $T$ is a coalition and $\mu^{\prime \prime}$ a matching that can be enforced over $\mu$ by $T$ such that:

(C1) $T \backslash S \neq \emptyset, S \backslash T \neq \emptyset$, and $T \cap S \neq \emptyset$;

(C2) $\mu^{\prime \prime}(i) \succeq_{i} \mu(i)$ for all $i \in T \backslash S$ and $\mu^{\prime \prime}(i) \succeq_{i} \mu^{\prime}(i)$ for all $i \in T \cap S$.

An objection $\left(S, \mu^{\prime}\right)$ against a matching is justified if there is no counterobjection against $\left(S, \mu^{\prime}\right)$.

Definition 2.6 The bargaining set is the set of matchings that have no justified objections.

Finally, a matching $\mu$ for a given marriage market $(M, W, P)$ is said to be weakly efficient if there is no other matching $\mu^{\prime}$ in which all agents are better off, i.e., $\mu^{\prime}(i) \succ_{i} \mu(i)$ for all $i \in N$.

\section{The set of weakly stable matchings}

In this section we study the structure of the set of weakly stable matchings. The following proposition is a straightforward consequence of the definitions of (weak) stability and the nonemptiness of the stable set (Gale and Shapley (1962)).

Proposition 3.1 Any stable matching is a weakly stable matching. Hence, the set of weakly stable matchings is non-empty.

That the concept of weakly stable matchings is indeed weaker than the concept of stable matchings follows from Example 3.2.

Example 3.2 Consider the marriage market (Knuth (1976)) $(M, W, P)$ where $M=\left\{m_{1}, m_{2}, m_{3}, m_{4}\right\}, W=\left\{w_{1}, w_{2}, w_{3}, w_{4}\right\}$, and the preferences $P=\left\{P\left(m_{1}\right), P\left(m_{2}\right), P\left(m_{3}\right), P\left(m_{4}\right), P\left(w_{1}\right), P\left(w_{2}\right), P\left(w_{3}\right), P\left(w_{4}\right)\right\}$ are given by

$$
\begin{aligned}
& P\left(m_{1}\right)=w_{1}, w_{2}, w_{3}, w_{4}, m_{1} \\
& P\left(m_{2}\right)=w_{2}, w_{1}, w_{4}, w_{3}, m_{2} \\
& P\left(m_{3}\right)=w_{3}, w_{4}, w_{1}, w_{2}, m_{3} \\
& P\left(m_{4}\right)=w_{4}, w_{3}, w_{2}, w_{1}, m_{4} \\
& P\left(w_{1}\right)=m_{4}, m_{3}, m_{2}, m_{1}, w_{1} \\
& P\left(w_{2}\right)=m_{3}, m_{4}, m_{1}, m_{2}, w_{2} \\
& P\left(w_{3}\right)=m_{2}, m_{1}, m_{4}, m_{3}, w_{3} \\
& P\left(w_{4}\right)=m_{1}, m_{2}, m_{3}, m_{4}, w_{4} .
\end{aligned}
$$


One can verify that there are ten stable matchings and two weakly stable matchings that are not stable. In the latter two matchings women $w_{1}, w_{2}, w_{3}$, and $w_{4}$ are matched to

$$
\begin{aligned}
& m_{1}, m_{3}, m_{2}, m_{4}, \quad \text { and } \\
& m_{4}, m_{2}, m_{3}, m_{1},
\end{aligned}
$$

respectively. Matching (1) is weakly stable because all its blocking pairs are weak: $\left(m_{2}, w_{1}\right) \rightarrow$ $\left(m_{3}, w_{1}\right) \rightarrow\left(m_{3}, w_{4}\right) \rightarrow\left(m_{2}, w_{4}\right) \rightarrow\left(m_{2}, w_{1}\right)$. Similarly, all blocking pairs for matching (2) are weak: $\left(m_{1}, w_{2}\right) \rightarrow\left(m_{4}, w_{2}\right) \rightarrow\left(m_{4}, w_{3}\right) \rightarrow\left(m_{1}, w_{3}\right) \rightarrow\left(m_{1}, w_{2}\right) . \diamond$

Next, we show that the set of weakly stable matchings does not have a lattice structure. Consider again the marriage market of Example 3.2. Let the women choose the best partner from matching (1) and the (stable) matching in which women $w_{1}, w_{2}, w_{3}$, and $w_{4}$ are matched to

$$
m_{2}, m_{4}, m_{1}, m_{3}
$$

respectively. The result is not a matching, since women $w_{1}$ and $w_{3}$ both choose man $m_{2}$. Hence, the set of weakly stable matchings does not have a lattice structure.

For the special class of neighbor marriage markets the sets of stable and weakly stable matchings coincide and are a singleton. A neighbor marriage market is a marriage market in which the agents are put along a line, alternating men and women and where only direct neighbors are possible acceptable partners.

Proposition 3.3 In a neighbor marriage market the sets of stable and weakly stable matchings coincide and are a singleton.

Proof. By the existence result of Gale and Shapley (1962) and Proposition 3.1 we are done if we show that there is at most one weakly stable matching. In fact, we will directly show which is the unique weakly stable matching.

It follows from individual rationality that for a matching $\mu$ to be weakly stable, there is no marriage in $\mu$ between two neighbors for which at least one of them prefers staying single to marrying the other agent. Nor can such a marriage serve as a blocking pair. This splits the marriage problem into several subproblems where every agent prefers marrying to any of his neighbors (note that there is only one neighbor if the agent is at an extreme of the line in the subproblem) to staying single. It is easy, but tedious, to check that for such kind of marriage market there is only one weakly stable matching. Combining the weakly stable matchings for the subproblems we obtain the unique weakly stable matching $\mu$ for the original problem.

\section{Weak stability and the bargaining set}

In this section we link the concept of weak stability with Zhou's (1994) bargaining set for the marriage model. Since we will make use of weak efficiency, we first present the following result on the relation of the stability concepts and weak efficiency. 
Proposition 4.1 Any stable matching is weakly efficient. There are, however, weakly stable matchings that are not weakly efficient.

Proof. One easily verifies that if a matching is not weakly efficient, then it is not individually rational or it has a blocking pair. In other words, if a matching is not weakly efficient then it is not stable.

To prove the second statement, consider the marriage market $(M, W, P)$ where $M=\left\{m_{1}, m_{2}, m_{3}\right\}, W=\left\{w_{1}, w_{2}, w_{3}\right\}$, and the preferences $P=\left\{P\left(m_{1}\right), P\left(m_{2}\right), P\left(m_{3}\right), P\left(w_{1}\right), P\left(w_{2}\right), P\left(w_{3}\right)\right\}$ are given by

$$
\begin{aligned}
& P\left(m_{1}\right)=w_{3}, w_{2}, w_{1}, m_{1} \\
& P\left(m_{2}\right)=w_{1}, w_{3}, w_{2}, m_{2} \\
& P\left(m_{3}\right)=w_{2}, w_{1}, w_{3}, m_{3} \\
& P\left(w_{1}\right)=m_{3}, m_{2}, m_{1}, w_{1} \\
& P\left(w_{2}\right)=m_{1}, m_{3}, m_{2}, w_{2} \\
& P\left(w_{3}\right)=m_{2}, m_{1}, m_{3}, w_{3} .
\end{aligned}
$$

Let $\mu$ be the matching defined by $\mu\left(m_{i}\right)=w_{i}$ for all $m_{i} \in M$, and let $\mu^{\prime}$ be the matching defined by $\mu^{\prime}\left(m_{1}\right)=w_{2}, \mu^{\prime}\left(m_{2}\right)=w_{3}$, and $\mu^{\prime}\left(m_{3}\right)=w_{1}$. Then, $\mu$ is a weakly stable matching; there are six blocking pairs and each of them is dominated by another one: $\left(m_{1}, w_{2}\right) \rightarrow\left(m_{1}, w_{3}\right) \rightarrow$ $\left(m_{2}, w_{3}\right) \rightarrow\left(m_{2}, w_{1}\right) \rightarrow\left(m_{3}, w_{1}\right) \rightarrow\left(m_{3}, w_{2}\right) \rightarrow\left(m_{1}, w_{2}\right)$. But $\mu$ is not weakly efficient since $\mu^{\prime}(i) \succ_{i} \mu(i)$ for all agents $i \in N$. This completes the proof.

For the example in the proof of Proposition 4.1 let $\mu_{M}$ denote the matching produced by the deferred acceptance algorithm of Gale and Shapley (1962) with men proposing; namely, $\mu_{M}\left(m_{1}\right)=w_{3}, \mu_{M}\left(m_{2}\right)=w_{1}$, and $\mu_{M}\left(m_{3}\right)=w_{2}$. Then, as one verifies readily, $\mu_{M}(m) \succ_{m}$ $\mu(m)$ for all $m \in M$ and $\mu_{M}(w) \succ_{w} \mu(w)$ for all $w \in W$. This implies that the decomposition lemma of Knuth (1976) does not hold for the set of weakly stable matchings. The decomposition lemma says that both sides of the market are in conflict on the set of stable matchings and it was a key result to show the lattice structure of the set of stable matchings.

Roth and Sotomayor (1990) showed that the core of a marriage market, which is the set of matchings that have no objection, coincides with the set of stable matchings. A result in the same vein for the bargaining set of a marriage market and the set of weakly stable matchings does not hold, because these sets do not always coincide. To see this, note that from the first condition in (C1) it immediately follows that an objection of the coalition $N$ cannot be counterblocked. Hence, the matching $\mu$ in the proof of Proposition 4.1 is not in the bargaining set. Since $\mu$ is weakly stable we conclude that the bargaining set does not coincide with the set of weakly stable matchings. Nevertheless, Theorem 4.2 shows that the bargaining set consists of the weakly stable matchings that are weakly efficient.

Theorem 4.2 In a marriage market, the bargaining set coincides with the set of weakly stable and weakly efficient matchings. 
Proof. We first prove that a matching that is not weakly efficient or not weakly stable cannot be contained in the bargaining set.

A matching that is not weakly efficient is not in the bargaining set because by $(\mathrm{C} 1)$ coalition $N$ has a justified objection. So, we are done if we prove that matchings that are not weakly stable are not in the bargaining set.

Let $\mu$ be a matching that is not weakly stable. We may assume that $\mu$ is individually rational (since otherwise $\mu$ is not in the bargaining set by $(\mathrm{C} 1)$, and we are done). Then, by definition of weak stability, there is a blocking pair that is not weak, say $(m, w)$. Let $S=\{m, w\}$ and let the matching $\mu^{\prime}$ be defined by $\mu^{\prime}(m)=w, \mu^{\prime}(w)=m$, and for $i \in N, i \neq m, w: \mu^{\prime}(i)=\mu(i)$ if $\mu(i) \neq m, w$ and $\mu^{\prime}(i)=i$ otherwise. Then, $\left(S, \mu^{\prime}\right)$ is an objection against $\mu$.

Now suppose there is a counterobjection, say $\left(T, \mu^{\prime \prime}\right)$, against the objection $\left(S, \mu^{\prime}\right)$. Then, since $S \backslash T \neq \emptyset$ and $S \cap T \neq \emptyset$, we have, without loss of generality, that $m \in T$ and $w \notin T$. Note that

$$
\mu^{\prime \prime}(m) \succeq_{m} w \succ_{m} \mu(m) \succeq_{m} m
$$

The relations in (4) follow from the counterobjection $\left(T, \mu^{\prime \prime}\right)$, the objection $\left(S, \mu^{\prime}\right)$, and the individual rationality of $\mu$, respectively. From (4) it follows that $\mu^{\prime \prime}(m) \neq m$. We will show that $\left(m, \mu^{\prime \prime}(m)\right)$ is a blocking pair that dominates $(m, w)$, contradicting that the blocking pair $(m, w)$ at $\mu$ is not weak. (This will complete the first part of the proof since then apparently the assumption that there is a counterobjection does not hold.) It suffices to prove that

$$
\begin{gathered}
\mu^{\prime \prime}(m) \succ_{m} \mu(m), \\
\mu^{\prime \prime}(m) \succ_{m} w, \text { and } \\
m \succ_{\mu^{\prime \prime}(m)} \mu\left(\mu^{\prime \prime}(m)\right) .
\end{gathered}
$$

Obviously, (5) follows immediately from (4).

We prove that (6) is true. Since coalition $T$ can enforce matching $\mu^{\prime \prime}$ over $\mu$ and since $\mu^{\prime \prime}(m) \neq \mu(m)$ (by (5)), it follows from (E) that $\left\{\mu^{\prime \prime}(m), m\right\} \subseteq T$. Then by $w \notin T, \mu^{\prime \prime}(m) \neq$ $w$. Hence, we conclude from $\mu^{\prime \prime}(m) \succeq_{m} w$ (by (4)) that $\mu^{\prime \prime}(m) \succ_{m} w$.

Finally, we prove that (7) holds. By now we know that $\mu^{\prime \prime}(m) \neq m, w$. So, from $S=$ $\{m, w\}$ it follows that $\mu^{\prime \prime}(m) \notin S$. We also noticed that $\mu^{\prime \prime}(m) \in T$. So, $\mu^{\prime \prime}(m) \in T \backslash S$. By (C2), $m=\mu^{\prime \prime}\left(\mu^{\prime \prime}(m)\right) \succeq_{\mu^{\prime \prime}(m)} \mu\left(\mu^{\prime \prime}(m)\right)$. Suppose that $m=\mu\left(\mu^{\prime \prime}(m)\right)$. Then, $\mu(m)=\mu^{\prime \prime}(m)$, contradicting (4). Hence, $m \neq \mu\left(\mu^{\prime \prime}(m)\right)$, and (7) follows.

To prove the other inclusion, let $\mu$ be a weakly stable matching that is weakly efficient. We will show that $\mu$ is contained in the bargaining set.

Suppose that a coalition $S \subseteq N$ has an objection $\left(S, \mu^{\prime}\right)$ against the matching $\mu$. We will show that there is a counterobjection $\left(T, \mu^{\prime \prime}\right)$ against $\left(S, \mu^{\prime}\right)$.

Note first that by weak efficiency of $\mu$ we have $S \neq N$. Furthermore, it follows from the individual rationality of $\mu$ and (E) that coalition $S$ consists of blocking pairs that are matched in $\mu^{\prime}$.

Now take an agent $i \in N \backslash S$. We may assume, without loss of generality, that $i \in M$. Say $i=m^{*}$. If there is a pair $(m, w)$ in $S$ matched in $\mu^{\prime}$ such that $m^{*} \neq \mu(w)$, then it is easily verified that, if $\# S>2$, then $\left(T, \mu^{\prime \prime}\right)$ with $T=\left\{m, w, m^{*}\right\}$ and $\mu^{\prime \prime}$ defined by $\mu^{\prime \prime}(m)=w$, 
$\mu^{\prime \prime}(j)=\mu(j)(j \neq m, w, \mu(m), \mu(w))$, and $\mu^{\prime \prime}(j)=j$ (if $j=\mu(m) \neq m$ or if $\left.j=\mu(w) \neq w\right)$ is a counterobjection against $\left(S, \mu^{\prime}\right)$. Now, suppose $\# S=2$. Then, $S=\{m, w\}$ for some blocking pair $(m, w)$ for $\mu$. Since $(m, w)$ is a blocking pair for $\mu$ and $\mu$ is weakly stable, there is another blocking pair $\left(m, w^{\prime}\right)$ or $\left(m^{\prime}, w\right)$ for $\mu$ that dominates $(m, w)$. Suppose there is a blocking pair $\left(m, w^{\prime}\right)$ for $\mu$ that dominates $(m, w)$. Note that $w^{\prime} \notin S$. One easily verifies that $\left(T, \mu^{\prime \prime}\right)$ where $T=\left\{m, w^{\prime}\right\}$ and $\mu^{\prime \prime}$ defined by $\mu^{\prime \prime}(m)=w^{\prime}, \mu^{\prime \prime}(j)=\mu(j)\left(j \neq m, w^{\prime}, \mu(m), \mu\left(w^{\prime}\right)\right)$, and $\mu^{\prime \prime}(j)=j$ (if $j=\mu(m) \neq m$ or if $j=\mu\left(w^{\prime}\right) \neq w^{\prime}$ ) is a counterobjection against $\left(S, \mu^{\prime}\right)$. Similarly, if there is a blocking pair $\left(m^{\prime}, w\right)$ for $\mu$ that dominates $(m, w)$, then $\left\{m^{\prime}, w\right\}$ has a counterobjection against $\left(S, \mu^{\prime}\right)$.

So, suppose that there is no pair $(m, w)$ in $S$ matched in $\mu^{\prime}$ such that $m^{*} \neq \mu(w)$. Then, for every pair $(m, w)$ in $S$ matched in $\mu^{\prime}$ we have $m^{*}=\mu(w)$. Hence, by the structure of $S$, the set $S$ consists of only one such a pair. The proof is completed following the same argument used in the preceding case to identify a counterobjection against $\left(S, \mu^{\prime}\right)$.

Remark 4.3 Consider once more the marriage market of Example 3.2. Let $\mu$ be the matching that matches women $w_{1}, w_{2}, w_{3}$, and $w_{4}$ with $m_{1}, m_{4}, m_{2}$, and $m_{3}$, respectively. Then, $\mu$ is weakly efficient (because $\mu$ matches for example $m_{1}$ to his most preferred woman), but $\mu$ is not weakly stable (since the blocking pair $\left(m_{2}, w_{1}\right)$ is not dominated by the only other blocking pair $\left(m_{2}, w_{4}\right)$ ). Now we can depict the relations between weak efficiency, (weak) stability, and the bargaining set in Figure 2. $\diamond$

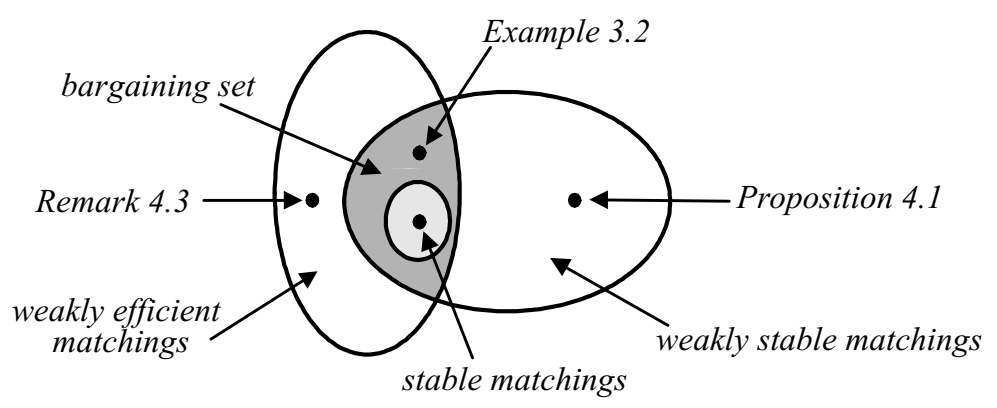

Figure 2: Relations between (weak) stability and the bargaining set

\section{Concluding remarks}

In this note we have introduced a relaxation of stability for the marriage model. The idea behind the concept is that we consider a matching to be stable if all blocking pairs are not credible, i.e., for each blocking pair one of its members can find a more attractive partner with whom he 
forms another blocking pair for the original matching. Obviously, the new concept can, with some adaptations, also be applied to more general settings of matching problems, for instance in many-to-one and many-to-many models. Thus, weak stability may also be of interest in view of the problem of the emptiness of the set of stable matchings in the models mentioned above with general preferences. Although we did not explore weak stability in other matchings models in this note, we think that it could be an interesting direction for further research.

Clearly, the bargaining set of Zhou (1994) is only one of the various bargaining sets that have been proposed since Aumann and Maschler (1964) started the study on bargaining. The bargaining set of Zhou (1994), however, has, in our opinion, several attractive features that previous solution concepts failed to satisfy. Nonetheless, it would still be interesting to see what happens if one applies the original bargaining set of Aumann and Maschler (1964) or the bargaining set of Mas-Colell (1989) to matching models. In this light, we would like to point out that in the proof of Theorem 4.2 we used all three conditions of (C1). It is precisely the combination of these conditions that was the novelty of the bargaining set of Zhou (1994). 


\section{References}

Aumann, R., and Maschler, M. (1964). "The Bargaining Set for Cooperative Games," in Advances in Game Theory, (M. Dresher, L. Shapley, and A. Tucker, Eds.). Princeton, NJ: Princeton University Press.

Gale, D., and Shapley, L. (1962). "College Admissions and the Stability of Marriage," Amer. Math. Monthly 69, 9-15.

Gärdenfors, P. (1975). "Match Making: Assignments Based on Bilateral Preferences," Behavioral Science 20, 166-173.

Knuth, D. (1976). Marriages Stables. Montreal: Les Presses de l’Université de Montreal.

Mas-Colell, A. (1989). "An Equivalence Theorem for a Bargaining Set," J. Math. Econ. 18, 129-139.

Roth, A., and Sotomayor, M. (1990). Two-Sided Matching: A Study in Game-Theoretic Modeling and Analysis. Econometric Society Monograph Series. New York: Cambridge University Press.

Zhou, L. (1994). "A New Bargaining Set of an N-Person Game and Endogenous Coalition Formation," Games Econ. Behavior 6, 512-526. 